\title{
A single technique to correct various degrees of upper lid retraction in patients with Graves' orbitopathy
}

\author{
Maarten Ph Mourits, Inna Vladimirovna Sasim
}

\begin{abstract}
Background-Several lengthening techniques have been proposed for upper eyelid retraction in patients with Graves' orbitopathy and variable rates of success have been reported. Most authors recommend different procedures for different degrees of retraction, but cannot prevent residual temporal retraction in a significant number of cases. The modified levator aponeurosis recession described by Harvey and colleagues, in which the lateral horn is cut completely, seems to be an exception to this rule, but was evaluated in a limited number of cases only.

Method-The authors further modified Harvey's technique by dissecting the aponeurosis together with Müller's muscle of the tarsus and the conjunctiva medially only to the extent necessary to achieve an acceptable position and contour of the eyelid in upright position. They also used an Ethilon 6.0 suture, instead of Vicryl, on a loop. It is placed between the tarsal plate and the detached aponeurosis to prevent spontaneous disinsertion. This modification was used in 50 Graves' patients (78 eyelids) with a upper lid margin-limbus distance ranging from 1 to $7 \mathrm{~mm}$ and evaluated using strict criteria.
\end{abstract}

Results-A perfect or acceptable result was obtained in 23 of 28 patients $(82 \%)$ with bilateral retraction and in 18 of 22 patients $(82 \%)$ with unilateral retraction. Seven eyelids were overcorrected (too low) and three undercorrected, necessitating reoperation. All other eyelids had an almond-like contour and a lid crease of 10 mm or less. No complications except subcutaneous haematomas were seen. Two patients showed a recurrence of lid retraction 9 months after the operation.

Conclusion-This technique is safe and efficacious and can be used for all degrees of eyelid retraction.

(Br F Ophthalmol 1999;83:81-84)

Upper eyelid retraction is a common symptom of Graves' orbitopathy. It causes a startled or sometimes aggressive appearance, ocular discomfort, and keratopathy. Immunosuppressive treatment such as orbital irradiation has been shown to be of little value in decreasing the eyelid aperture. ${ }^{1}$ Surgery, therefore, is required to correct eyelid retraction, but there is no agreement as to the best surgical technique. In the past, we have used sclera as a spacer in lengthening the upper lids, but we found the results to be unpredictable. ${ }^{2}$ We have now developed a levator complex disinsertion technique that closely resembles Harvey and colleagues' modification of levator aponeurosis recession. ${ }^{3}$ This procedure is relatively easy to perform and can be used for any degree of upper eyelid retraction. In the present paper, we describe the technique and the results of 78 such procedures performed in 50 patients.

\section{Methods}

INDICATIONS

Indications for upper eyelid lengthening are keratopathy, ocular discomfort, and/or cosmetic rehabilitation. Eyelid surgery is not performed until the patient is clinically and biochemically euthyroid, has reached the quiescent stage of the orbitopathy and all signs and symptoms of Graves' disease have been stable for at least 6 months. (An exception is made for patients with corneal ulcers, but they are not included in this study.) If orbital decompression and/or strabismus surgery are necessary, these operations are done first.

\section{SPECIFIC PREOPERATIVE EXAMINATION}

The eyelid aperture and the lid margin-limbus distance are measured in the primary position of gaze. Slit lamp examination is performed to detect corneal staining. Photographs of the eyes are taken before and approximately 3 months after the eyelid lengthening procedure.

\section{PREOPERATIVE PREPARATION}

Half an hour before the operation starts, the patient is given $1000 \mathrm{mg}$ paracetamol and 10 mg oxazepam to prevent excessive pain and anxiety before, during, and after surgery. Two drops of oxybuprocaine $0.4 \%$ and of Tetracaine (amethocaine) $0.5 \%$ are administered twice to both eyes. The patient is seated in an armchair that can be placed in full supine position electronically. The eyelids are cleaned and disinfected with a $0.5 \%$ cetrimide solution and an $80 \%$ dehydrated alcohol solution and the upper lid crease of both eyes is marked with a marking pen. Approximately $4 \mathrm{ml}$ of prilocaine $1 \%$ together with adrenaline $1 / 200000$ per eyelid is injected subcutaneously. (Patients with cardiac disorders are given prilocaine $1 \%$ only.) Before injection, the prilocaine solution is warmed to $37^{\circ} \mathrm{C}$ to reduce injection discomfort. The entire face of the patient is then disinfected with the above mentioned solutions and draped, leaving the forehead, eyes, cheeks, nose, and lips uncovered in such a way that
Accepted for publication 30 July 1998 
there is no traction of the drapes on the eyelids. The patient is put in upright position to evaluate the effect of the anaesthetic injection on the position of the eyelids.

SURGICAL TECHNIQUE

Back in supine position, a $30 \mathrm{~mm}$ incision through skin and orbicularis muscle is made along the mark. The lower part of the incision is carefully pulled downwards with a one toothed hook by the assistant and the upper part of the tarsal plate is dissected free with a pair of Westcott scissors. The orbital septum is opened with the same scissors and the preseptal fat bulges out. Only excessive fat is excised after careful cautery. A Desmarres retractor elevates the upper half of the wound and the preseptal fat, allowing a clear view on the levator aponeurosis and tarsal plate (Fig 1a). Adhesions between the aponeurosis and the upper orbital margin, if present, are cut. Haemostasis is achieved using bipolar cautery. The lateral horn of the levator is identified and cut completely with sharp scissors along the medial side of the lacrimal gland. In severe cases, the incision of the aponeurosis is continued superiorly as far as the lateral part of Whitnall's ligament. Starting from the cut edge, the levator together with Müller's muscle is stripped from the conjunctiva and the lateral part of the tarsal plate using blunt scissors, carefully avoiding the lacrimal gland lobules (Fig 1b). At this stage it may be necessary to inject some anaesthetic solution between the conjunctiva and Müller's muscle in order to ease the dissection procedure and to minimise pain and bleeding. The detached part of the aponeurosis and Müller's muscle retract upwards and medially, creating a triangle of uncovered conjunctiva with the base down. Now, the upper lid "comes down". The effect of the dissection is checked repeatedly by removing the instruments and asking the patient to open his or her eyes and look up and down. If the position of the eyelid seems acceptable, it is also checked in the upright position. As a further drop of the upper lid averaging $1.5 \mathrm{~mm}$ can be expected when the adrenaline has worn off, the ideal position of the upper lid at this moment is at the corneal limbus. If there is still residual retraction, the dissection of the aponeurosis together with Müller's muscle is continued medially until an acceptable position has been reached. (In extreme cases, this requires dissection as far as the medial horn.) A 6.0 nylon suture is placed through the upper horizontal middle of the tarsal plate and sutured on a loop to the detached aponeurosis (Fig 1C). If required by the upper lid contour, a second suture on a loop is placed laterally to the first. The patient is then placed in upright position again for a final check. Adjustments are made to the tension of the sutures to fix the eyelid contour and position. The skin is closed with 6.0 nylon sutures. No bandage is applied. Dexamytrex eye ointment is put on the cornea and "ice-glasses" are put on the eyes for 30-45 minutes. The sutures are removed after 5 days. The procedure takes about 30 minutes per eyelid.
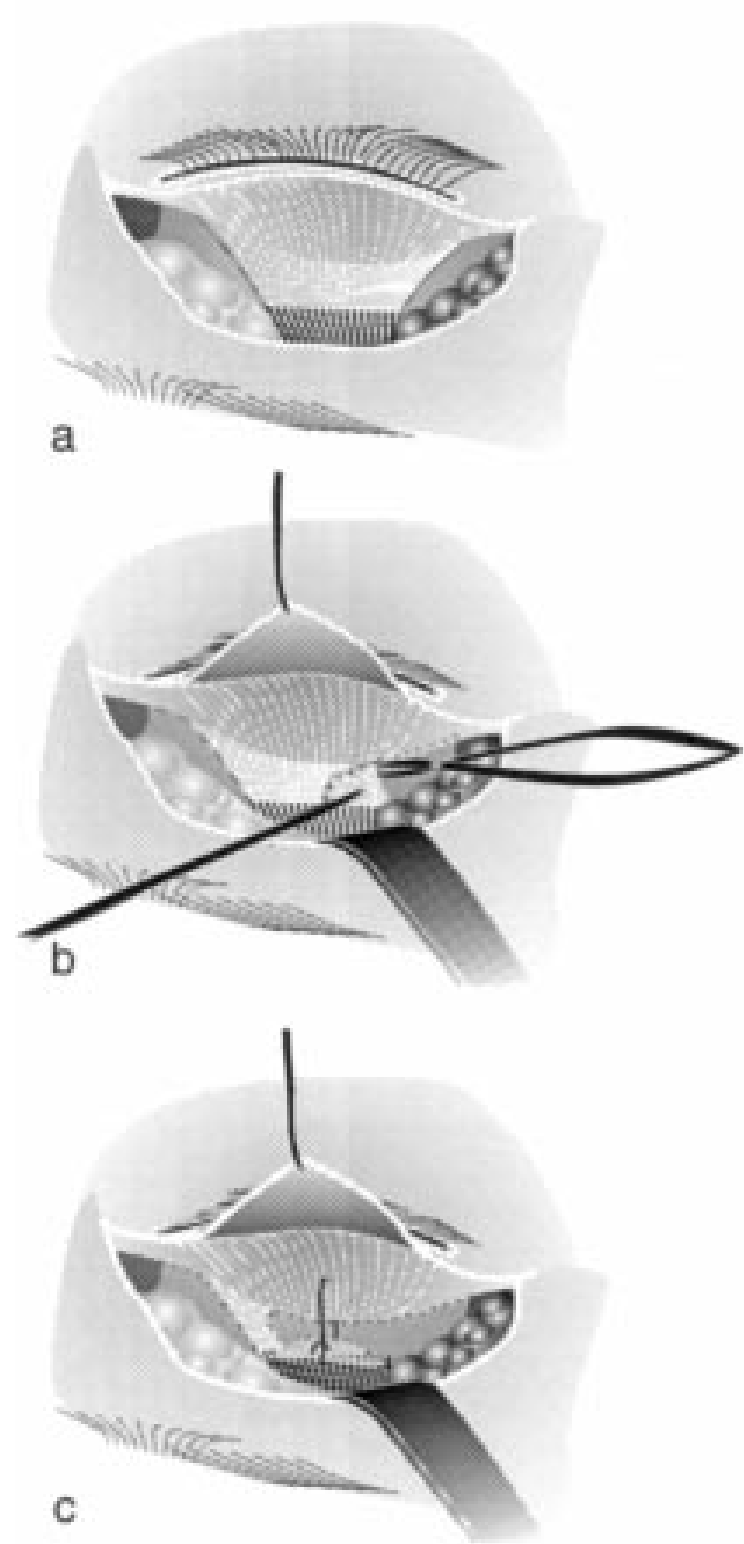

Figure 1 (a) View of the tarsal plate, the aponeurosis, and levator muscle after opening of the orbital septum. In the lower corner on the right, part of the lacrimal gland is visible. (b) The lateral horn is cut; the aponeurosis together with Müller's muscle is stripped from the conjunctiva and the upper border of the tarsal plate. (c) A 6.0 nylon suture on a loop is placed between the tarsal plate and the partly detached levator aponeurosis and Müller's muscle to prevent further spontaneous disinsertion.

POSTOPERATIVE EVALUATION

The outcome of surgery was evaluated 3 months after the operation by measuring the eyelid aperture and the lid margin-limbus distance in the primary position. Corneal staining was assessed with a biomicroscope. The patient was asked to classify the subjective result as completely satisfactory, satisfactory, or unsatisfactory. Pre- and postoperative photographs were compared. The outcome was classified as perfect, acceptable, or unacceptable (Table 1). To assess the long term results, the patients were again evaluated approximately 9 months after surgery. 


\section{Table 1 Criteria for evaluation of upper lid lengthening in Graves'orbitopathy}

Perfect result:

1 The upper 0.5 to $1.5 \mathrm{~mm}$ of the cornea in the 12 o'clock position is covered by the eyelid.

2 The difference in lid aperture between the left and right side is $\leqslant 1 \mathrm{~mm}$.

3 The patient is completely satisfied.

4 The lid margin contour is smooth.

5 The lid crease is within $7-10 \mathrm{~mm}$ from the lid margin.

6 The skin folds of the right and left lid are symmetrical.

Acceptable result:

1 The upper eyelid margin is within $0.5 \mathrm{~mm}$ of the limbus, or covers no more than $2 \mathrm{~mm}$ of the cornea in the 12 o'clock position.

2 The difference in lid aperture between the left and right side is less than $2 \mathrm{~mm}$.

3 The patient is satisfied and demands no further surgery.

4-6 As in a perfect result.

Unacceptable result:

One or more of the above mentioned criteria are not fulfilled.

\section{Results}

Between January 1995 and December 1996, 78 upper eyelids (of 50 consecutive patients) were lengthened. All patients were operated by one of us (MPM). There were 47 women (mean age 46 years, range 26-76 years) and three men (mean age 57 years, range 35-71 years). All patients were euthyroid and had stable Graves' orbitopathy. Previous treatments included methylprednisolone pulse therapy because of dysthyroid optic neuropathy in two patients, oral prednisone therapy in seven patients, orbital irradiation in 14 patients, coronal three wall decompression in 13 patients, translid two wall decompression in nine patients, strabismus surgery in 10 patients, and blepharoplasty (skin and fat removal) in eight patients. The indication for lid surgery was punctate keratitis in 20 patients, ocular discomfort (need for lubricants, photophobia, easy tearing) in 17 patients, and pure cosmetic rehabilitation in 13 patients.

Twenty eight patients had bilateral upper lid retraction ranging from 1 to $7 \mathrm{~mm}$ (average, $2.96 \mathrm{~mm}$ ). Bilateral upper lid lengthening resulted in a perfect result in $14(50 \%)$ (Fig 2), in an acceptable result in nine (32\%) (Fig 3), and in a unacceptable result in five patients $(18 \%)$ (Fig 4). In these five patients, five eyelids were too low, one eyelid was still too high, two were perfect, and two acceptable. Approximately 9 months after the operation, 27 patients showed the same position of their upper lids as 6 months earlier; one patient, however, had bilateral retraction again requiring surgery for cosmetic rehabilitation.

Unilateral lid lengthening in 22 eyelids (mean retraction $2.41 \mathrm{~mm}$, range $1-4 \mathrm{~mm}$ ) resulted in 11 perfect results (50\%) (Fig 5), seven acceptable results (32\%), and four unacceptable results $(18 \%)$. Of these four eyelids, two were too low and two still too high. In this group also, one patient had a recurrent upper lid retraction 9 months after lid lengthening.

Three months postoperatively, in all 78 lids operated, a perfect result per eyelid was seen in 44 lids (56\%), an acceptable result in 24 lids $(31 \%)$ and an unacceptable results in 10 lids $(13 \%)$. Over- and undercorrected eyelids were seen in both young and old individuals and in patients with a history of mild and severe orbitopathy. No relation with previous treatment modalities was found.
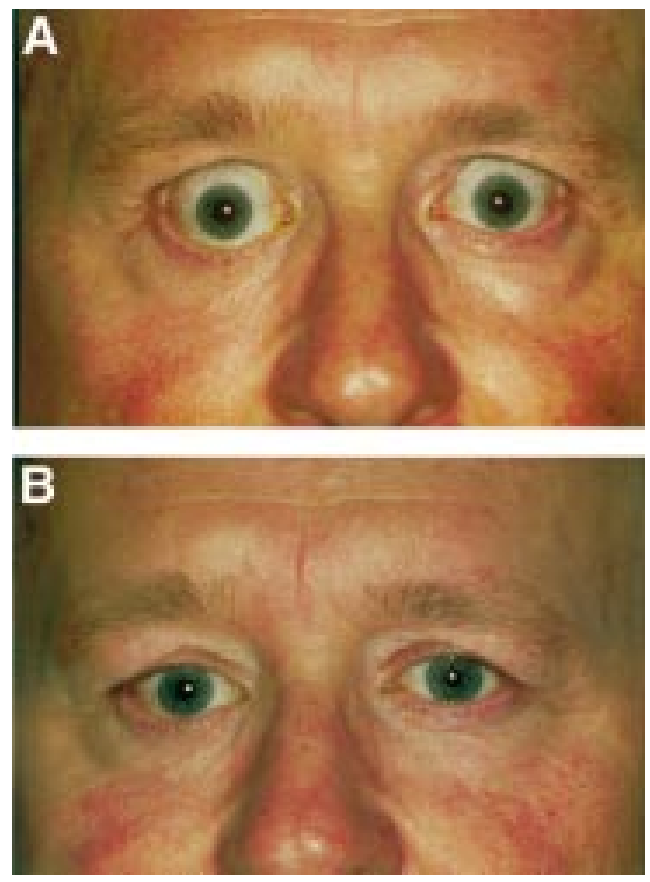

Figure 2 Patient with bilateral upper lid retraction before (A) and after (B) correction. Perfect result. Good position, good contour. Left-right asymmetry less than $1 \mathrm{~mm}$.
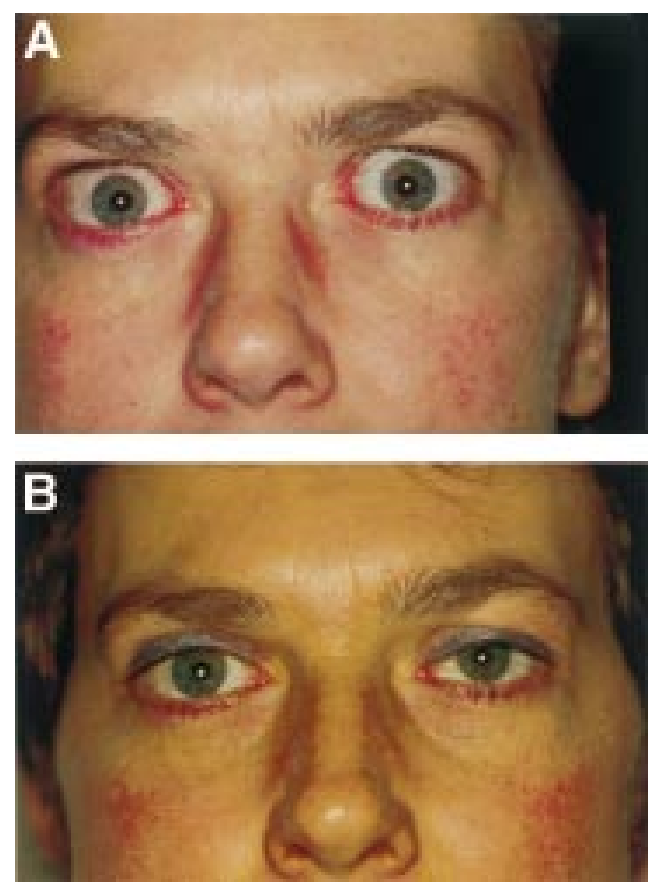

Figure 3 Patient with bilateral upper lid retraction before (A) and after (B) correction. Acceptable result. Good position, good contour. Left-right asymmetry more than 1 but less than $2 \mathrm{~mm}$.

\section{Discussion}

The aim of correction of upper lid retraction in patients with Graves' orbitopathy is disappearance of keratopathy, complete relief of ocular discomfort, and complete restoration of preillness appearance. A technique is required that sets and maintains the height and the contour of an eyelid within an accuracy of $1 \mathrm{~mm}$. The effects of local anaesthetics, perioperative haemorrhages, wound healing, Hering's law, and even of changed postoperative orbicularis 

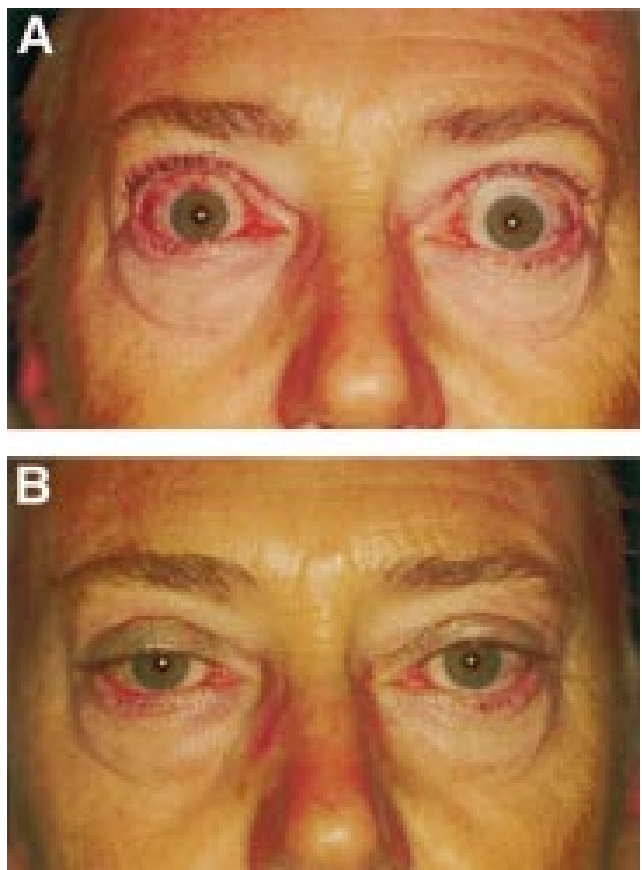

Figure 4 Patient with bilateral upper lid retraction before (A) and after (B) correction. Overcorrection right upper lid.
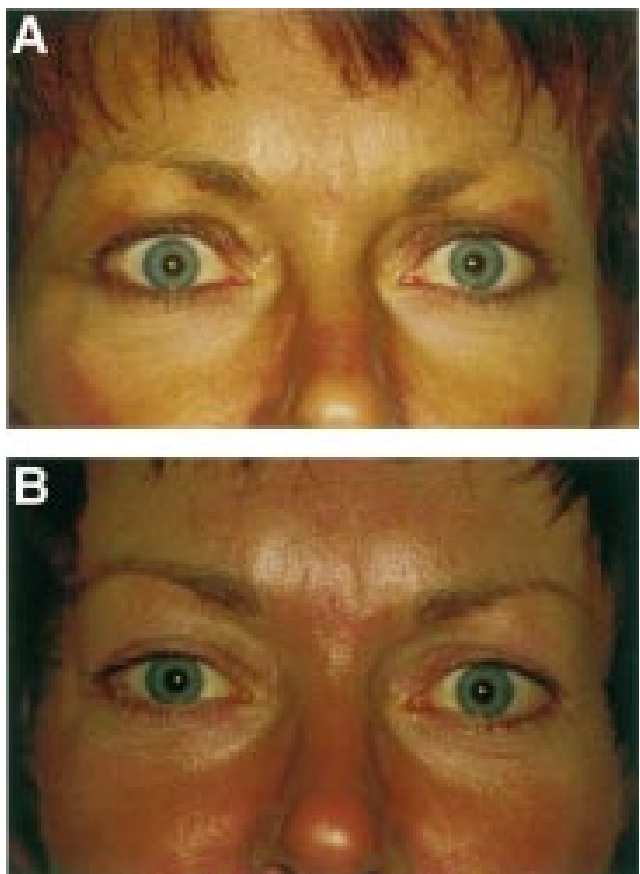

Figure 5 Patient with unilateral upper lid retraction before (A) and after (B) correction. Perfect result. Good position, good contour. Left-right asymmetry less than $1 \mathrm{~mm}$.

and frontalis tonus may annul the efforts. Therefore, it is not surprising that many procedures have been described and that surgeons keep on refining their techniques..$^{3-8}$ Upper lids can be lengthened by an anterior or a posterior approach, by "Z-myotomy" by excision or recession of Müller's muscle, by levator recession with or without a spacer and with or without adjustable sutures, and by levator transposition. ${ }^{10}$ Examples of successful results of all techniques have been reported, but it is difficult to assess which is the best procedure for a large group of patients with different degrees of retraction. This is further complicated by the lack of general agreement on a definition of success. Using strict criteria, this series shows that a single technique is satisfactory in different grades of retraction.

Since temporal retraction is the most important aspect of upper lid retraction in patients with Graves' orbitopathy, we consider cutting the lateral horn of the aponeurosis to be a sine qua non in lengthening the upper lid. We have seen no patients with complications as a result of doing this, no matter how much or how little retraction they had. Overcorrection was due to dissecting the aponeurosis and Müller's muscle too far medially. This is best evaluated with the patient in an upright position. If this happens, the aponeurosis must be sutured back to the tarsus. Even when the eyelid position is fine, a suture- knot on a loop-is necessary to prevent further spontaneous disinsertion when the patient opens and closes the eyes again. This suture should be non-absorbable, as we have seen (before this study started) a number of secondary overcorrections 3-4 weeks after the use of Vicryl. Apparently, Vicryl dissolves before wound healing has progressed far enough to prevent secondary disinsertion. Using nylon sutures in levator lengthening, we have seen no complications such as corneal erosion, but we have always been careful to leave a layer of conjunctiva between the suture and the cornea.

To our surprise, we had two patients in this series with a recurrence of upper lid retraction 6-9 months after eyelid lengthening. These patients were still euthyroid and showed no other signs of reactivation of their Graves' orbitopathy. Their eyelid positions were at a perfect height immediately and 3 months postoperatively. On reoperating these patients, we found considerable fibrosis. A matter for further investigation is whether levator lengthening can be accompanied by fat excision. In our series, failures were often patients in whom fat had been excised as well, but the numbers were too small for statistical analysis. Modest fat excision is sometimes unavoidable and probably safe to do. However, excision of larger amounts of fat and skin should be postponed to a next operation.

1 Prummel MF, Mourits MP, Blank L, et al. Randomised double-blind trial of prednisone vertsus radiotherapy in Graves' ophthalmopathy. Lancet 1993;342:949-54.

2 Mourits MP, Koornneef L. Lid lengthening by sclera interposition for eyelid retraction in Graves' ophthalmopathy. $\mathrm{Br}$ position for eyelid retraction in

3 Harvey JT, Corin S, Nixon D, et al. Modified levator aponeurosis recession for upper eyelid retraction in Graves' aponeurosis recession for upper eyelid retractic

4 Putterman AM, Urist M. Surgical treatment of upper eyelid retraction. Arch Ophthalmol 1972;87:401-5.

5 Putterman AM. Surgical treatment of thyroid-related upper eyelid retraction. Ophthalmology 1981;88:507-12.

6 Harvey JT, Anderson RL. The aponeurotic approach to eyelid retraction. Ophthalmology 1981;88:513-24.

7 Thaller VT, Kaden K, Lane CM, et al. Thyroid lid surgery. Eye 1987;1:609-14.

8 Tucker SM, Collin R. Repair of upper eyelid retraction: a comparison between adjustable and non-adjustable sutures. Br 7 Ophthalmol 1995;79:658-60.

9 Henderson JW. Relief of eyelid retraction. Arch Ophthalmol 1965;74:205-16.

10 Ceisler EJ, Bilyk JR, Rubin PA, et al. Results of Müllerotomy and levator aponeurosis transposition for the correction of upper eyelid retraction in Graves' disease. Ophthalmology 1995;102:483-92. 